\title{
Durées de la mue, de l'intermue des adultes, et longévité de Stenasellus virei Dollfus Crustacé Asellote troglobie).
}

\author{
Par Guy Magniez ${ }^{11}$
}

Avec planches 111 (1)-112 (2)

Comme tous les Crustacés, les Sténaselles rejettent périodiquement la partie externe, plus ou moins calcifiée et inextensible de leur cuticule: ils muent.

Les mues peuvent être l'occasion d'un accroissement sensible des dimensions générales, surtout pour les individus jeunes, en cours de croissance. Elles peuvent s'accompagner de processus de morphogenèse, portant sur certains des appendices, avant la puberté. Elles permettent aussi à la régénération de s'effectuer à la suite d'ablation de portions plus ou moins importantes des appendices. Elles peuvent être liées à des phases importantes du cycle reproducteur, telles que la fécondation et la ponte, dans le cas des femelles adultes. Elles continuent enfin d'affecter les individus adultes âgés, dont le taux de croissance est devenu très faible, en dehors même de tout cycle de reproduction.

\section{A. Mue des Isopodes: cas général}

En règle générale, le rejet de l'exuvie des Isopodes s'effectue en deux temps: une ligne de rupture transversale apparaît dans la membrane articulaire qui sépare les péréionites IV et $\mathrm{V}$ et l'animal quitte son exuvie postérieure comme un doigt de gant, en quelques instants. La mue postérieure correspond donc aux trois segments postérieurs du péréion et au pléotelson (TAIT, 1917).

La mue antérieure libère de leur ancienne cuticule la région céphalique, le segment des maxillipèdes et les quatre péréionites antérieurs avec leurs appendices correspondants. L'animal s'en débarasse d'une seule pièce également. Cette deuxième phase d'exuviation survient en général quelques heures après la précédente, mais la durée de la période de repos intermédiaire est fonction de la température ambiante (UNWIN 1920, MAERCKS

1) Laboratoire de Biologie Animale et Générale, Faculté des Sciences, 6 Boulevard Gabriel, 21 Dijon, France. 
1930; VANDEL 1947) et aussi de la taille des individus dans une espèce donnée: elle tend à s'accroître pour les spécimens âgés (BALESDENT 1964).

B. Déroulement de la mue dans le cas des espèces de STENASELLUS étudiées

\section{Données bibliographiques:}

Dans sa monographie de Stenasellus, Racovitza (1950) cite l'observation d'un unique individu qui venait de subir sa mue antérieure. L'auteur ajoute, sans en fournir la preuve «La région postérieure était encore contenue dans l'ancienne carapace». En effet, il eût été nécessaire, pour appuyer cette affirmation, de conserver l'animal en vie jusqu'à ce que se produise la mue postérieure elle-même, ce qui ne fût pas le cas. L'apport de Racovitza est donc ici extrêmement fragmentaire. L'auteur poursuit «Cette observation démontre que Stenasellus mue de la même façon qu'Asellus aquaticus», or toutes les observations relatives à cet Aselle, de même qu'à d'autres espèces du genre, sont concordantes: la mue des Aselles se produit en deux temps. La portion postérieure du corps, comprenant les trois derniers péréionites et le pléotelson en premier lieu, puis la portion antérieure. Cette seconde phase du phénomène est retardée par rapport à la première de quelques heures à deux jours, mais elle dépend de plusieurs facteurs, en particulier de la température des eaux, de l'âge des Aselles utilisés (BALESDENT, 1964), du sexe de ceux-ci et aussi de l'espèce en cause. Ce dernier auteur apporte des observations très détaillées: sur plusieurs centaines de mâles ayant mué, certains ont d'abord perdu l'exuvie antérieure, sans que le départ de l'exuvie postérieure ait été retardé pour des raisons uniquement mécaniques. Il s'agit donc d'une véritable inversion, sans conséquences pour la biologie de l'Aselle, puisque la mue des mâles adultes est une simple crise de croissance et n'interfère pas avec les processus de reproduction.

Aucune inversion n'a encore été signalée en ce qui concerne les femelles adultes. Dans ce cas, un certain nombre de mues, dites mues parturielles, présentent une grande importance, car elles sont liées à l'accouplement, à la fécondation et à la ponte: la mue parturielle se produit alors que la chevauchée nuptiale est déjà commencée et le départ de l'exuvie postérieure libère les orifices génitaux femelles fonctionnels sur les coxopodites des péréiopodes V. La fécondation interne peut alors s'effectuer. L'exuvie antérieure, étant rejetée ensuite, libère les oostégites fonctionnels, qui forment le marsupium. La partie postérieure de celui-ci masque les orifices génitaux, si bien que les oeufs qui sont alors pondus progressivement tombent dans la cavité incubatrice. Le mécanisme de la mue doit être ici étroitement synchronisé avec les processus physiologiques de la fécondation et de la ponte, ainsi qu'avec les phases du comportement des deux animaux. Une inversion des mues postérieure et antérieure, en particulier, rendrait la fécondation impossible, faute d'orifices génitaux femelles fonctionnels. 
C'est à Husson (1957), que sont dues les premières observations suivies sur la biologie et en particulier sur le phénomène de mue de St. virei. Elles furent réalisées, cette fois, sur des animaux en élevage, aussi bien à la grotte de Moulis, qu'au Laboratoire où les Crustacés furent observés pendant près de deux ans (de septembre 1955 à juillet 1957). L'auteur pût alors mettre en évidence que l'accouplement et la ponte des Asellotes hypogés (aussi bien Asellus cavaticus Leydig que Stenasellus virei Dollfus), peuvent se faire normalement dans les conditions d'élevage au laboratoire, alors qu'il est assez difficile de les obtenir avec les Amphipodes du genre Niphargus. A maintes reprises, des femelles d'A. cavaticus devinrent ovigères. Pour St. virei, 3 femelles devinrent ovigères au bout d'un an d'installation dans le bac d'élevage. La première difficulté dans l'étude du phénomène de la mue, note l'auteur, résulte de la présence dans le récipient du limon argileux qui constitue le substratum habituel de l'animal. Il est nécessaire, pour rendre possibles des observations précises, de réaliser des élevages en aquarium sans argile. Les exuvies peuvent alors être recueillies et observées facilement puisque, dans ce cas, aucune particule limoneuse ne peut se coller à leur surface.

Dans ces conditions, les Sténaselles élevés au laboratoire souterrain ont fourni à l'auteur des mues en deux temps dans la plupart des cas. Cependant, le déroulement du phénomène pendant la nuit à empêché de vérifier la succession réelle des deux temps. Husson, ayant relevé le caractère anormal de la référence de Racovitza (1950) à la mue d'Asellus aquaticus, souligne le caractère exceptionnel de la perte de l'exuvie antérieure en premier lieu.

Dans les élevages réalisés au laboratoire de Sarrebrück, une série d'observations ont par contre prouvé que $S t$. virei pouvait muer à la manière citée par RACOVITZA, la partie antérieure du corps perdant d'abord son exuvie. Une planche photographique illustre ainsi cette première phase du phénomène.

De plus, l'auteur décrit trois cas particuliers, au cours desquels l'exuviation s'est faite en un seul temps, comme le montre une série de clichés. Dans ces cas particuliers, la mue d'une seule pièce pourrait rappeler celle des Amphipodes.

Pourtant, ceux-ci quittent leur ancienne carapace par une ligne de déhiscence dorsale, à la limite entre prosome et métasome, alors que les exuvies entières de Stenasellus étudiées par l'auteur avaient été quittées ventralement par les Isopodes. A la suite de ses observations répétées, l'auteur est amené à conclure que, si Stenasellus virei subit, dans la plupart des cas, une mue en deux temps (l'exuviation antérieure précédant la postérieure), il peut parfois abandonner son exuvie en une seule fois. 


\section{Observations personnelles dans le milieu naturel:}

A plusieurs reprises, nous avons remarqué dans les stations les plus populeuses de St. virei, telles que les grottes du Mont de Chac (Haute-Garonne) et de l'Estelas (Ariège), qui permettent d'observer simultanément plusieurs dizaines de Sténaselles, un individu présentant une différence d'intensité de la coloration rose entre la moitié postérieure et l'antérieure. C'est vraisemblablement un tel cas qui fut observé en premier lieu par Racovitza. Nous n'avons jamais encore pu trouver les exuvies abandonnées par ces Crustacés dans les flaques argileuses où ils vivent, soit parce qu'elles sont indiscernables du substrat, en raison des particules de limon qui s'y collent, soit parce que la plupart restent dans les galeries, soit enfin parce qu'elles seraient dévorées par les Crustacés après la mue. Ce sont les élevages entretenus pendant quelques cinq années qui permettent d'apporter une importante contribution à la connaissance du phénomène.

\section{Observations personnelles de la mue:}

Parmi les récipients d'élevage, certains ne furent garnis que de sable ou de feuilles mortes macérées, à l'exclusion de toute argile de grotte, conformément à la technique indiquée par Husson (1957). Ce sont ces élevages qui ont permis d'observer le phénomène le plus commodément. En effet, les mues sont toujours difficiles à repérer dans les récipients dont le fond est revêtu d'une épaisse couche d'argile noyée sous un à deux centimètres d'eau: les Sténaselles y trouvent des conditions qui rappellent leurs biotopes naturels, creusent de nombreux terriers et s'y réfugient périodiquement, ce qui s'oppose à leur observation continue. De plus les exuvies peuvent y rester indécelables, souillées qu'elles sont, en général, de particules minérales.

Avant sa mue, l'animal montre une teinte rose délavée caractéristique de l'espèce. La coloration rose est fournie par un pigment rouge dissous dans l'hémolymphe qui semble imprégner aussi la musculature. Cette coloration évoque celle connue chez certains Isopodes terrestres comme Androniscus dentiger Verhoeff.

Lorsque le Crustacé se débarrasse, en quelques dizaines de minutes, de son exuvie postérieure, dont il sort (orifice de rupture situé entre les péréionites IV et V) en opérant des tractions au moyen des quatre paires de péréiopodes antérieurs, il apparaît revêtu d'une nouvelle cuticule absolument transparente. La teinte rose du pigment interne est alors beaucoup plus visible de l'extérieur, elle semble donc plus intense, d'autant qu'aucune particule étrangère ne s'est encore fixée sur la portion du corps qui vient de muer. L'exuvie postérieure, qui comprend les trois péréionites postérieurs et le pléotelson, avec leurs appendices et leurs armatures de soies, conserve en général sa cohésion même si des déchirures latérales sont fréquentes, de part 
et d'autre de la ligne de déhiscence. Elle est relativement solide et sa teinte blanchâtre explique comment la teinte rose des animaux en période d'intermue se trouve comme diluée par la présence d'un tégument normal blanchâtre relativement opaque. Notons aussi que l'exuvie de l'intestin postérieur fait partie intégrante de la mue postérieure. L'intestin postérieur de St. virei s'étend très loin vers l'avant, puisque le niveau d'insertion des deux grands caecums entériques se trouve à la hauteur du premier péréionite (St. virei ne possède qu'une paire de coecums entériques bien développés, la seconde étant rudimentaire, caractère qui le distingue des Asellides vrais).

Au bout de 24 à 48 heures environ, le Crustacé a retrouvé un aspect roseblanchâtre uniforme et il faut attendre à peu près une dizaine de jours, dans le cas général, pour que se produise la seconde phase d'exuviation.

La mue de la région antérieure quitte alors le corps, également à la façon d'un doigt de gant. L'animal en sort en s'aidant par des mouvements de recul provoqués par les trois paires postérieures de péréiopodes. La libération des antennes et des pièces buccales est souvent laborieuse et si la plupart des individus s'en acquittent en quelques dizaines de minutes, elle demande jusqu'à 48 heures à certains autres. La mue antérieure est, de ce fait, généralement très chiffonnée et parfois déchirée sur la face sternale. Elle est également plus mince et plus transparente que la postérieure, ayant sans doute supporté une résorption plus poussée. Le Stenasellus qui vient de quitter son exuvie antérieure montre alors une région antérieure rose-vif très transparente qui permet d'observer plus facilement les canaux déférents du mâle ou les ovaires de la femelle, mais, 24 à 48 heures plus tard, il retrouve l'aspect habituel de l'espèce.

\section{Discussion:}

Nos observations semblent ainsi marquer une certaine discordance avec celles de nos prédécesseurs. En fait, celle-ci est plus apparente que réelle.

En ce qui concerne les observations de Husson (1957) qui sont fondées, non pas sur l'examen d'un individu unique, comme ce fût le cas pour Racovitza (1950), mais sur des groupes d'adultes élevés au laboratoire, une série de clichés montre la réalité du phénomène décrit. Les individus étudiés par les deux auteurs semblent pourtant être des mâles. Dans les élevages expérimentaux réalisés, il est de pratique courante de disposer d'un nombre de mâles adultes très supérieur au nombre des femelles, afin d'accroître les chances de fécondation de chacune d'elles. Comme les mues successives de mâles seraient légèrement plus rapprochées les unes des autres que celles des femelles adultes, on peut supposer que les observations faites porteraient vraisemblablement sur les premières et non sur les secondes Or, Balesdent (1964), a montré que les mâles $d^{\prime}$ A. aquaticus $L$. dans un 
certain pourcentage des cas, étaient sujets à une inversion des phases de la mue. La succession mue postérieure-mue antérieure n'est donc qu'une réalité statistique et non un impératif absolu pour le mâle de cette espèce. Il en est vraisemblablement de même pour le mâle de $S t$. virei, et nous pouvons admettre qu'au moins dans certaines conditions, le phénomène atteint une fréquence particulière.

Le cas des femelles est différent. Nous avons rappelé l'importance que revêt la succession précise des phases de leurs mues parturielles en liaison avec les processus reproducteurs. Pas plus les centaines d'Aselles femelles étudiés par BALESDENT (1964), que les quelques 200 Sténaselles femelles observées par nous, n'ont montré de cas d'inversion.

\section{Evaluation de la durée du phénomène:}

Nous n'envisageons ici que les mues des adultes, c'est-à-dire celles des mâles ayant terminé la différenciation sexuelle de leurs premiers et seconds pléopodes et celles des femelles acquérant leurs oostégites (à la base des maxillipèdes et des quatre paires antérieures de péréiopodes) et les conservant, sous une forme variable, au cours des exuviations suivantes.

Si les observations ont porté sur la totalité des élevages réalisés et s'étendent sur plusieurs années, certains cas de mue comportent une indétermination de la durée du phénomène. Ce sont celles qui eurent lieu dans les récipients d'élevage à substrat argileux dans lesquels une proportion notable des Sténaselles sont toujours enfouis, quel que soit le moment de la journée durant lequel a lieu l'observation. Ces cas ont servi cependant à établir des vérifications. Les Sténaselles élevés sur des substrats nonargileux sont observés quotidiennement. Lorsque, de loin en loin, un cas de mue se déclenche, l'animal en cause est immédiatement isolé, avec un peu de son eau d'origine, dans un petit cristallisoir individuel, garni de sable et suivi avec soin.

a) Cas des mâles: toutes les mues successives sont identiques. La durée totale de l'exuviation, comprise entre la perte de la mue postérieure et celle de la mue antérieure, est en moyenne de 13 à 14 jours, les animaux étant placés dans une eau à température normale constante de $11^{\circ}$. Ces valeurs sont fournies par des mâles adultes de 6,5 à $7 \mathrm{~mm}$ provenant des stations classiques dont on peut obtenir un matériel suffisamment abondant (Mont de Chac, l'Estelas). Cette durée s'abaisse parfois à 12 jours, mais n'atteint que très rarement 15 jours.

b) Cas des femelles:

b1) Les femelles d'aspect juvénile subissent, lorsqu'elles atteignent une taille de 6 à 7,5 mm (variable selon les stations d'origine et les individus) une mue particulière au cours de laquelle apparaissent les oostégites. Dans 
un élevage réalisé dans ce but, cette mue donnait, dans $90 \%$ des cas des femelles adultes à oostégites rudimentaires (petites languettes subquadrangulaires ne se recouvrant pas et n'atteignant pas le plan médian), dites $q$ 1; mais dans les $10 \%$ des cas restants, elle donnait des femelles à grands oostégites lamelleux formant un marsupium, dites $q$ 2. La durée de cette mue est comprise entre 8 et 11 jours.

b 2) Les femelles à oostégites rudimentaires ( +1$)$ peuvent subir des mues dont elles sortent identiques à elles-mêmes, mis à part un très léger accroissement de taille. Les oostégites restent alors «non fonctionnels». On peut les qualifier de mues de repos sexuel. Le déterminisme de la succession de ces mues reste obscur. Cette succession n'a pas été observée dans la nature, mais sur des femelles élevées isolément et sans doute en état d'inanition plus ou moins marqué. Les durées notées pour ces mues s'échelonnent de 8 à 13 jours.

b 3) Plus fréquemment les femelles à oostégites rudimentaires subissent une mue dite parturielle, car liée à la reproduction. La perte de l'exuvie postérieure est accompagnée de la formation d'orifices génitaux fonctionnels à la base des péréiopodes $\mathrm{V}$, qui permettront la fécondation interne et ultérieurement la ponte.

La perte de l'exuvie antérieure est, dans ce cas, suivie de l'apparition des oostégites fonctionnels qui se croisent médialement et se recouvrent pour former la poche incubatrice dans laquelle les oeufs sont pondus et se développeront. Dans un cas particulier, nous avons noté un écart de 14 jours et 23 heures entre la mue postérieure et la mue antérieure. Les valeurs extrêmes observées sont 10 jours et 16 jours et la moyenne 14 jours.

b 4) Lorsque les femelles ont libéré leurs jeunes, elles conservent leur marsupium vide durant quelques mois. Il faut noter, au passage, que cette phase ne dure que quelques jours pour Asellis aquaticus (BALESDENT, 1964) et 4 à 11 jours seulement pour le troglobie A. cavaticus Leydig (HenRY, 1965). Ces femelles subissent ensuite une mue après laquelle les oostégites reprennent leur aspect de petites lamelles isolées, non fonctionnelles. Comme les femelles déjà ovigères lors de leur capture, aussi bien que celles qui le sont devenues en élevage, ont été isolées afin de recueillir leur descendance, les mues de ce type sont les plus nombreuses (103 cas de 1961 à 1965). La durée de ces mues est dans le cas général de 10 à 11 jours. Nous citerons, à titre d'exemples extrêmes, une période de 17 jours, pour une femelle de très grande taille et, à l'opposé, un cas tout à fait isolé, qui montra les deux phases de la mue survenant à 24 heures d'intervalle.

\section{Cas d'autres espèces de STENASELLUS:}

Les données sont extrêmement fragmentaires.

a) Stenasellus buili Remy, 1949. 
Nous n'avons pu nous procurer qu'une quinzaine d'individus vivants de cette espèce dont il n'existe toujours qu'une seule station connue, celle de Soulatgé (Aude, France). Deux cas de mue, intéressant 2 individus différents, conduisent à admettre que la mue, en deux parties, la postérieure, puis l'antérieure, comme c'est le cas pour nos observations de St. virei, exige une durée supérieure à 10 jours, tandis que l'intermue serait très long, notablement supérieur à 1 an, pour les grandes femelles de 14 à $15 \mathrm{~mm}$.

b) Stenasellus breuili Racovitza, 1924:

Cette espèce n'était connue que par 2 individus adultes; un mâle et une femelle au repos génital, récoltés, en 1919, par Bolivar, Breuil et JeANNEL à la Cueva Akelar (Navarra) et à la Cueva de Aitzquirri (Guipuzcoa). Une unique femelle fut prise par STAMmer, en 1935, dans la seconde des deux grottes. Les 3 exemplaires furent fixés dès leur capture et aucune observation biologique ne fât alors possible.

En 1966, nous avons pu, avec R. Rouch, capturer vivants 5 mâles et 2 femelles adultes à la Cueva Akelar. Placés en élevage pendant 6 mois, nous n'avons pu enregistrer qu'un cas d'exuviation. Le mâle de St. breuili en cause a subi sa mue en deux temps, comme les St. virei et buili. La durée entre les pertes des exuvies postérieure et antérieure fut comprise entre 10 et 15 jours pour cet individu de $9 \mathrm{~mm}$.

\section{Comportement de l'animal en cours de mue}

\section{Pendant les phases d'exuviation:}

Lorsqu'il se débarrasse de l'une ou de l'autre des demi-exuvies, l'individu manifeste, pendant quelques dizaines de minutes, une grande activité musculaire utilisant les péréiopodes non mis en cause pour s'arc-bouter au substratum, position qui lui permet de se dégager de son exuvie. On note également que le déplacement des segments du péréion les uns sur les autres facilite cette opération. Le passage du Crustacé à l'intérieur de ses terriers facilite, le cas échéant, le glissement vers l'arrière de la mue postérieure.

\section{Entre les deux phases d'exuviation:}

Pendant la dizaine de jours que dure la mue, les individus jeunes, ainsi que les mâles adultes, montrent une tendance très nette à un isolement, au cours duquel leur activité devient quasi-nulle. Régulièrement ces animaux quittent l'eau, montent sur les parois verticales émergées de leur récipient et risquent de s'échapper. C'est pourquoi les récipients d'élevage, généralement en verre, sont eux-mêmes placés dans des boites en plastique fermées, garnies d'un peu d'eau, où sont recueillis les fugitifs éventuels ${ }^{1}$.

1) Cette précaution est surtout indispensable pour les élevages en enceinte isotherme, dont l'atmosphère est très éloignée du degré de saturation et dans laquelle les Sténaselles sortant de l'eau mourraient très vite par dessiccation. Néanmoins, pour les élevages poursuivis dans la grotte-laboratoire de Moulis, dont l'air est saturé, elle évite la fuite, donc la perte des animaux en observation. 
Les femelles en cours de mue, elles, ne semblent que très rarement réagir de cette façon, comportement pouvant peut-être se justifier par les nécessités de la reproduction.

\section{Fragilité des Sténaselles en cours de mue:}

a) Dans les récipients où sont groupés mâles et femelles adultes, en vue d'obtenir la reproduction, ces individus sont réunis, pour des raisons d'encombrement, avec une densité supérieure à celle du milieu d'origine. La fréquence des rencontres y est donc plus élevée. Dans ces conditions, nous avons constaté, à plusieurs reprises, des cas de cannibalisme. Les femelles, dont la taille dépasse celle des mâles de $2 \mathrm{~mm}$ environ et qui sont nettement plus robustes, sont capables de s'attaquer à un mâle qui vient de perdre l'une des demi-exuvies.

Au cas où l'individu attaqué n'a pu se mettre à l'abri dans son terrier ou sous une pierre, elles entament la moitié du corps qui n'est plus protégée par une carapace indurée.

Assez curieusement, les tendances carnassières des femelles semblent se manifester plus ou moins fréquemment et intensément, suivant leur origine. Les populations provenant du gouffre de Padirac (Lot) ou de Couflens de Betmajou (Ariège), paraissent particulièrement agressives ${ }^{2)}$.

b) Pour St. virei, la phase qui sépare les rejets de l'une et de l'autre des demi-exuvies correspond à une période de fragilité générale. En élevage la mortalité des individus adultes est particulièrement forte à ce moment. Il est fréquent que la mort des individus âgés survienne sans que la mue antérieure ait pu s'accomplir. Les exuvies de certains adultes âgés se détachent difficilement et seulement par lambeaux. De plus, ces individus sortent de l'épreuve amputés de portions de divers appendices (péréiopodes, fouet de l'antenne ou de l'antennule, etc. ...).

\section{Mue et régénération naturelle des appendices}

Les Sténaselles récoltés présentent parfois des portions d'appendices qui sont absentes: partie distale du fouet de l'antennule ou plus souvent de l'antenne, parfois un ou plusieurs articles d'un péréiopode.

L'appendice peut faire presque complètement défaut: péréiopode rompu dans la région proximale du basipodite, correspondant à une ligne privilégiée d'autotomie, uropodes cassés au ras du corps, au niveau de la partie antérieure du protopodite.

2) Cette question de comportement sera reprise ultérieurement. 


\section{Régénération normale:}

L'animal amputé présente un cal cicatriciel brun au niveau de la section. A la mue qui suit l'accident (souvent 9 à 12 mois après, puisque la mue elle-même et les jours qui suivent sont particulièrement favorables à de telles amputations), l'appendice retrouve une structure normale. Dans le cas du fouet de l'antenne, le nombre d'articles est réduit, mais il se complète lors de la mue suivante par l'adjonction d'articles intermédiaires. Le péréiopode régénère directement en présentant, dès le début, le nombre définitif d'articles; cependant la taille de l'appendice est inférieure à celle de son symétrique et la régulation totale n'est atteinte qu'à la seconde mue. Les uropodes retrouvent immédiatement une structure biramée, mais présentent une taille inférieure de moitié à la normale; la mue suivante rétablit leurs dimensions définitives.

La régénération s'étend donc sur une période de 18 à 24 mois. Nous avons observé jusqu'à 5 appendices mis en cause simultanément sur un mâle adulte du gouffre de Padirac ( 3 péréiopodes manquants, 1 péréiopode amputé au carpopodite et le fouet de l'antenne gauche sectionné en son milieu), sans que la vitalité de l'animal soit atteinte. La première mue a donné des péréiopodes plus courts que les normaux; les dactylopodites de petite taille ne portaient encore que l'épine sternale distale. Il est vraisemblable que l'épine proximale ne serait apparue qu'à la seconde mue, pour les individus pourvus typiquement de deux épines dactyliennes.

\section{Cas d'hétéromorphose:}

a) Un mâle adulte de $7 \mathrm{~mm}$ environ, récolté par VIRE, au puits de Padirac, en janvier 1913 (collections du Muséum National d'Histoire Naturelle), possédait le péréiopode VII droit coupé au niveau du tiers distal du basipodite, la section étant obturée par un cal cicatriciel, La partie basale du basipodite avait bourgeonné, en direction caudale, un régénérat hétérotypique comportant: d'une part, l'ébauche d'un appendice ambulatoire à quatre articles, dont le troisième laisse deviner une structure de méropodite (le propodite et dactylopodite sont donc absents) et, d'autre part, une lamelle subquadrangulaire orientée vers l'arrière, garnie de tiges marginales lisses, qui présente l'aspect d'une rame exopodiale de premier pléopode mâle (appendice du segment immédiatement postérieur (fig. 1, A B).

b) Une femelle juvénile de 5,5 mm, récoltée en 1965 à Couflens de Betmajou (Ariège), dont le pléopode II droit, au lieu de présenter l'aspect de lamelle triangulaire uniarticulée, est formé de deux lamelles identiques, à la taille près, (l'antérieure étant la plus petite), unies par la ligne d'insertion, et formant entre elles un dièdre de $45^{\circ}$ environ, (fig. $1 \mathrm{C}$ ). 
c) Enfin, il convient de rappeler pour mémoire, le cas des femelles anormales, à aspect externe intersexué, signalées antérieurement (MAGNiEz, 1963). Sur ces femelles âgées, il apparaît, sur le pléonite I, tout d'abord des moignons, puis des appendices identiques aux premiers pléopodes du mâle. L'évolution de ces appendices doit se dérouler au cours de plusieurs mues successives, donc sur plusieurs années de la vie de l'adulte et parfois même après un cycle de reproduction.

\section{E. Intermue et longévité de STENASELLUS VIREI}

\section{Durée de l'intermue:}

Dans une population naturelle, la découverte d'un animal en cours de mue est un évènement rare, l'unique observation de RACovitza en fait foi. Lorsque des adultes sont capturés dans une station, nous ignorons à quelle époque remonte leur dernière mue. La première mesure possible est donc celle qui sépare la date de leur capture de la première exuviation observée.

a) Sur un groupe de 35 femelles adultes, pourvues d'oostégites rudimentaires, provenant de plusieurs stations, 8 ont mué après 6 mois environ d'élevage, 13 après 9 mois et 14 après 10 mois. Ces premiers chiffres donnent une idée de l'espacement possible des mues.

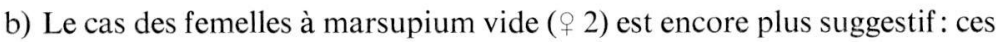
femelles ont élevé une portée de jeunes, les ont libérés et attendent la mue régressive de leurs oostégites. Citons une récolte simultanée de 31 d'entre elles, dont, au bout de 6 mois, aucune n'avait encore subi cette mue.

Si l'on ajoute qu'après la mue parturielle, une durée d'environ 10 mois sépare la ponte de la libération du dernier jeune du marsupium ${ }^{1)}$, la durée qui sépare une mue parturielle de la mue régressive peut-être déjà considérée comme supérieure ou égale ă 16 mois. Quant aux nombreuses femelles qui ont accompli, au laboratoire, un cycle de reproduction complet, l'intervalle entre ces deux mues est de l'ordre de 15 à 16 mois, les extrêmes étant 12 et 18 mois.

c) La durée d'intermue des mâles adultes est de l'ordre de 9 à 12 mois, donc très comparable, quoique un peu inférieure, à celle des femelles au repos sexuel. Des individus de l'un et de l'autre sexe, présumés très âgés, ont été conservés 16,17 et 19 mois sans présenter d'exuviation. Ici encore, les variations paraissent être liées au lieu d'origine des animaux étudiés et peut-être aussi aux conditions artificielles des élevages.

1) Cette question fera ultérieurement l'objet d'une étude détaillée. 


\section{Longévité de STENASELLUS VIREI:}

Nous ne pouvons encore aborder ce problème directement, c'est-à-dire en suivant de bout en bout les diverses phases de la vie d'un même groupe de Sténaselles du même âge. En effet:

a) La découverte d'individus juvéniles dans les stations naturelles est un événement rarissime. Il est donc impossible d'utiliser actuellement un tel matériel comme base d'une expérience de longévité.

b) Les jeunes $S t$. virei nés au laboratoire en 1961 sont donc les plus anciens exemplaires dont nous connaissions l'âge avec exactitude. Or, aucun des jeunes individus de sexe femelle ne dépasse aujourd'hui une taille de $6 \mathrm{~mm}$ (un peu plus de $4 \mathrm{~mm}$ pour les mâles); aucun d'eux n'est encore adulte après 66 mois (cinq ans et demi) d'existence.

c) Des individus récoltés à l'état adulte aux grottes de Sainte-Catherine (Ariège), qui mesuraient alors entre 7 et $8 \mathrm{~mm}$, ont été conservés vivants pendant 62 mois (plus de cinq ans!), sans qu'aucune mortalité anormale-ne se soit encore manifestée. Ils sont toujours en observation, ainsi que ceux du cas suivant.

d) Une femelle, récoltée dans les mêmes conditions que celles du lot précédent, est devenue ovigère après 58 mois de captivité, ce qui représente une vie adulte virtuelle minimale de 73 mois (plus de six ans, en tenant compte de la durée globale du cycle reproducteur en cours). D'autres femelles, de 7 à $8 \mathrm{~mm}$, après avoir accompli un cycle de reproduction (15 mois), sont demeurées au repos génital depuis 3 ans. Examinées à l'occasion de leur dernière mue, elles montrent par transparence, dans leurs ovaires des ovocytes en voie de croissance. La maturation de ces ovocytes exige donc au moins une nouvelle période d'intermue, au cours de laquelle doit s 'achever leur croissance ( 9 mois environ) et à laquelle doit succéder un second cycle de reproduction ( 15 mois environ); ce qui permet d'estimer la durée minimale de la vie de ces femelles à l'état adulte à 75 mois. Cette longévité est donc du même ordre que celle des précédentes, qui dépasse également six ans. Il se pourrait d'ailleurs que certaines femelles de très grande taille (11 à $12 \mathrm{~mm}$ à la grotte du Goueil di Her) aient à leur actif un troisième cycle de reproduction, ce qui ajouterait au total cité plus haut deux années supplémentaires d'existence à l'état adulte.

e) En confrontant ces divers résultats, se rapportant essentiellement à des Sténaselles qui continuent d'être suivis en élevage, on peut estimer la durée de vie de Stenasellus virei à 10 ans ou plus (11 ans et 9 mois, pour les femelles précitées, qui sont des individus de taille tout à fait banale et non des cas exceptionnels). 
En ce qui concerne les mâles auxquels les critères précédents ne peuvent s'appliquer, il est également possible de leur attribuer une longévité normale de 10 ans, en tenant compte des observations poursuivies sur les jeunes nés au laboratoire et sur les adultes capturés en grottes.

\section{Comparaison avec certains Asellotes épigés:}

Les divers travaux réalisés sur les Asellides d'eau douce (A. aquaticus, $P$. meridianus, etc.), établissent que le cycle vital complet de ces Crustacés épigés se déroule normalement au cours d'une même année, même si leur vie se poursuit occasionnellement sur deux années consécutives.

Leur puberté survenant environ deux mois après l'éclosion à la belle saison, leur vie n'excède jamais un an. La durée globale de vie de l'espèce hypogée peut donc être estimée à peu près à dix fois celle des formes épigées de taille similaire ( $A$. aquaticus, par exemple).

Les durées respectives des mues et des intermues, dans ces deux espèces, paraissent être approximativement dans le rapport de 1 à 10 et tendent même à le dépasser, surtout pour les individus âgés. Rappelons que les deux demi-mues des Aselles épigés sont espacées de quelques heures, un jour au plus et que les intermues durent 16 jours en moyenne à $10^{\circ}$, en période de repos sexuel, mais 30 jours en période d'incubation ( $A$. aquaticus, d'après BALESDENT, 1964). La croissance et la reproduction sont donc extrêmement ralenties dans la forme troglobie, par rapport à ce que montrent les espèces épigées.

\section{Comparaison avec d'autres Crustacés cavernicoles:}

Daum (1954), estime que les durées d'intermue des Sphaeromiens cavernicoles Caecosphaeroma burgundum Dollfus, sont de l'ordre de 12 à 17 mois. La gestation dans le marsupium exige quant à elle de 11 à 12 mois, ce qui permet d'attribuer à cet Isopode une longévité d'au moins dix ans. Notons l'étroite similitude entre les observations de Daum portant sur le Sphaeromien et les nôtres relatives à l'Asellote pyrénéen. Cette similitude se retrouve jusque dans l'importance variabilité de la durée des intermues des deux Crustacés.

Ginet (1960), dans la grande monographie qu'il a consacrée au Niphargus virei Chevreux, parvient à des conclusions très semblables en comparant les durées d'intermue et les longévités de l'Amphipode troglobie à celles des Gammariens épigés. L'énorme accroissement de la longévité et de la durée des intermues est une caractéristique de toutes les espèces cavernicoles, lorsqu' on les compare aux formes épigées les plus voisines. Stenasellus virei n'échappe donc pas à cette règle qui paraît s'appliquer à nombre de formes installées depuis fort longtemps dans le domaine hypogé. 


\section{F. Mue et formes phorétiques}

Les Sténaselles servent de support à divers organismes. Citons Choanophrya stenaselli Matjasic, 1963, trouvé sur St. virei dans de nombreuses stations des grottes des Pyrénées. Les Acinétiens existent aussi sur d'autres espèces européennes (St. hungaricus Mehely, St. breuili Racovitza, entre autres), ainsi que sur les Metastenasellus congolais, comme nous avons pu le constater.

Déjà RAcovitzA (1950) signalait que ces organismes phorétiques «Acinétiens et Bactériacées moniliformes» envahissaient la carapace de St. virei, d'une manière extrêmement variable, selon les stations. Dans certain cas des Vorticellides pullulent sur les individus de cette espèce maintenus en élevage. Lorsque les ovules d'une femelle n'ont pas été fécondés, ils s'altèrent rapidement dans le marsupium de celle-ci; en une quinzaine de jours, ils sont envahis par le mycélium d'un champignon encore indéterminé, si bien que la cavité incubatrice finit par être occupée par un peloton dense de filaments mycéliens qui peuvent même déborder les oostégites et s'étendre jusqu'aux régions pleurales du péréion. La mue permet, dans ce cas, à la femelle (ou à l'individu porteur dans le cas général) de se débarrasser périodiquement de ces organismes étrangers qui, lorsqu'ils sont nombreux et se fixent sur les endopodites respiratoires des pléopodes III, IV et V, gênent considérablement les échanges avec le milieu. Le développement des organismes phorétiques demeure relativement lent à l'obscurité, c'est-à-dire dans le milieu naturel du Crustacé. Il n'en est plus de même lorsque St. virei est élevé en lumière intermittente ou continue. Dans ces conditions des formes épizoïques nouvelles et surtout des épiphytes chlorophylliens apparaissent. Ces organismes se développent rapidement et se multiplient sur la carapace. Nous avons observé des cas d'envahissement par des Chlorelles et des Algues vertes filamenteuses. Ces Algues se multiplient de préférence au niveau des pléopodes respiratoires, sans doute par suite du rejet continu du gaz carbonique métabolique à ce niveau. Leur présence entraîne un trouble des mouvements respiratoires de l'hôte. Placés dans les mêmes conditions, les Asellides épigés, qui muent beaucoup plus fréquemment, tous les 20 jours, en moyenne, parviennent à se débarrasser des organismes phorétiques avant toute prolifération excessive, mais St. virei, dont le rythme des mues, extrêmement ralenti n'est pas sensiblement modifié en milieu éclairé, se trouve gravement handicapé et incapable d'effectuer la régulation nécessaire.

\section{G. Conclusions générales}

Les précisions apportées ici, sur la durée de vie de St. virei concordent avec les observations poursuivies sur d'autres Crustacés cavernicoles Elles vérifient à nouveau une régle générale qui s'applique à de nombreux 
groupes animaux dont des représentants vivent dans les grottes (Vandel, 1964). La tendance à l'allongement de la durée de vie observée jusqu'ici, tant sur des Invertébrés que sur des Vertébrés cavernicoles, se manifeste dans ce cas avec une netteté particulière.

L'allongement de la vie de St. virei ne résulte pas d'un accroissement du nombre des mues et des intermues, mais seulement d'un extrême allongement de périodes d'intermues. Cependant les mues elles-mêmes sont soumises au ralentissement que montrent tous les processus biologiques des cavernicoles. La division en deux temps de l'exuviation des Isopodes est particulièrement favorable à la mise en évidence de ce phénomène.

St. virei et les autres espèces du genre étaient surtout connus par les descriptions spécifiques et la monographie de Racovitza (1950), mais les données relatives à leur biologie étaient rares (Husson, 1957). C'est grâce à des élevages poursuivis pendant plus de cinq années qu'il a été possible d'apporter quelques précisions sur le cycle vital de ce Crustacé hypogé dont la durée de la vie dépasse de beaucoup celle des Asellides épigés. La connaissance précise et complète de la biologie des Sténaselles nécessite une prolongation des observations sur de longues durées, à la mesure même de la longévité de ce très ancien cavernicole.

dècembre 1967

\section{RÉSUMÉ}

Des observations personnelles portant sur de nombreux cas de mue de l'Asellote hypogé Stenasellus virei Dollfus, 1897, semblent montrer que le phénomène se produit en deux temps. La perte de l'exuvie antérieure est séparée de celle de l'exuvie postérieure par une période de 8 à 16 jours, dans le cas des adultes des populations cavernicoles pyrénéennes et cantabriques de Stenasellus virei. Les intermues durent de 9 à 18 mois pour ces mêmes individus. Il faut admettre pour cette espèce, une durée de vie minimale de 10 ans.

Des valeurs du même ordre de grandeur sont prévisibles pour les deux espèces pyrénéennes: Stenasellus breuili Racovitza, 1924 et Stenasellus buili Remy, 1949.

Ces observations concordent avec celles qui ont été réalisées précédemment sur d'autres Crustacés Péracarides cavernicoles, tels que Caecosphaeroma burgundum Dollfus et Niphargus virei Chevreux, par Daum (1954), Husson (1959) et Ginet (1960).

\section{SUMMARY}

The author's observations of numerous cases of molting in the hypogean asellid Stenasellus virei Dollfus, 1897 appear to show that the phenomenon occurs in two steps. The loss of the anterior exuvium is separated from that of the posterior exuvium by a period of 8 to 16 days in adults from cavernicolous populations of Stenasellus virei in the Pyrenees and Cantabrian Alps. The intermolts last from 9 to 18 months for the same individuals. One must allow, for this species, a minimun life span of 10 years. Values of the same order of magnitude are anticipated for the two other Pyrenees species, Stenasellus breuili Racovitza, 1924 and Stenasellus buili Remy, 1949. These observations agree with those previously made on other cavernicolous peracarid crustaceans, such as Caecosphaeroma burgundum Dollfus and Niphargus virei Chevreux, by Daum (1954), Husson (1959) and GiNET (1960). 


\section{BIBLIOGRAPHIE}

BALESDENT, M. L. (1964) - Recherches sur la sexualité et le déterminisme des caractères sexuels d'Asellus aquaticus Linné (Crustacé Isopode). Th. Fac. Sc. Univ. Nancy, $1-231$.

Charniaux-Legrand, H. (1952) - Le cycle d'intermue des Amphipodes et ses particularités chez les formes terrestres (Talitridae). Arch. Zool. exp. gen. Paris, $88,4,178-204$.

Daum, J. (1954) - Zur Biologie einer Isopodenart unterirdischer Gewässer: Coecosphaeroma (Vireia) burgundum Dollfus. Ann. Univ. Saraviensis, III, 1/2, $104-159$.

Drach, P. (1944) - Etude préliminaire sur le cycle d'intermue et son conditionnement hormonal chez Leander serratus (Pennant). Bull. Biol. France-Belgique, Paris, 78, 40-62.

Ginet, R. (1958) - Observations sur la biologie de Niphargus (Amphipodes Gammaridés troglobies). Sous le Plancher, Dijon, 6, 44-46.

- (1960) - Ecologie, éthologie et biologie de Niphargus (Amphipodes Gammaridés hypogés). Ann. Spéléol. Paris, 15, 127-376.

- (1966) - Influence de la nutrition sur la durée de la vie des poecilothermes. Ann. Biol. Paris, 5, 1/2, 75-93.

Haemmerli-Boveri, V (1926) - Über die Determination der secundären Geschlechtsmerkmale (Brutsackbildung der weiblichen Wasserassel durch das Ovar). Zeitschr. $f$. wergl. Physiol. Berlin, 4, 668-698.

Henry, J. P. (1965) - Contribution à l'étude de la biologie d'Asellus cavaticus Leydig. Intern. Journ. Speleol. Lehre, 1, 3, 279-286.

- (1965) - Remarques sur le cycle biologique d'Asellus cavaticus Leydig (Crustacé Isopode troglobie). Actes $4^{\circ}$ Cong. Intern. Spéléol. Ljubljana (sous presse).

HuEt, E. (1883) - Nouvelles recherches sur les Crustacés Isopodes. Th. Fac. Sci. Univ. Dijon, 9, 1-142.

Husson, R. (1957) - A propos de la biologie du Crustacé troglobie aquatique Stenasellus virei Dollfus. Ann. Univ. Saraviensis, 6, 74, 8259-8269.

- (1959) - Les Crustacés Péracarides des eaux souterraines. Considérations sur la biologie de ces cavernicoles. Bull. Soc. Zool. France, 84, 4, 219-231.

- (1962) - Les ressources alimentaires des animaux cavernicoles. Cahiers d'Etudes biologiques, Lyon, 8/9, 103-116.

Jeannel, R. et Racovitza, E. G. (1929) - Enumération des grottes visitées, 1918 au 1927, (7e série, Biospeologica LIV), Arch. Zool. exp. gen. Paris, 68, 2, $293-608$.

Juchault-Stoll, C. (1964) - La mue en deux temps chez Paragnathia formica (Hesse). Arch. Zool. exp. gén., 104, 1-22.

Maerks, H. (1930) - Sexualbiologische Studien an Asellus aquaticus L. Zool. Anz. Leipzig, 48, 399-508.

Magniez, G. (1963) - A propos d'une anomalie de certaines femelles de Stenasellus virei Dollfus (Crustacé Isopode troglobie). Ann. Spéléol. Paris, 18, 4, $483-490$.

- G. (1966) - Contribution à la systématique des Stenasellinae d'Afrique (Crustacés, Asellotes). Intern. Journ. Speleol., Lehre, 2, 1/2, 173-190.

Matjašic, J. (1963) - Une nouvelle Choanophrya (Ciliata, Suctoria) sur Stenasellus virei. Ann. Spéléol., Paris, 18, 3, 267-270.

Messner, B. (1965) - Ein morphologisch-histologischer Beitrag zur Hautung von Porcellio scaber (Latr) und Oniscus asellus (L) (Isopoda terrestria). Crustaceana, 9, $288-301$.

Needham, A. E. (1942) - Micro-anatomical studies on Asellus. Quart. Journ. Micr. Sci. G. B., 184, 1, 49-72.

Racovitza, E. G. (1950) - Asellides première série: Stenasellus. Arch. Zool. exp. gen. Paris, $87,1-94$.

Remy, P. (1948) - Sur quelques Crustacés cavernicoles d'Europe. Notes biospéol. Paris, 3, 35-47. 
- (1949) - Stenasellus buili n. sp. de la grotte de la Giraudasso à Soulatgé Aude. Bull. Soc. linn. Lyon, 18, 7, 153-157.

Stammer, H. J. (1936) - Eine neue Höhlenwasserassel aus Spanien, Stenasellus buchneri und die Verbreitung der Gattung Stenasellus. Zool. Anz. Leipzig, 114, $137-141$.

TAIT, J. (1917) - Experiments and observations on Crustacea: part. II, Moulting of Isopods. Proc. R. Soc. Edinburgh, 37, 59-68.

Unwin, E. E. (1920) - Notes upon the reproduction of Asellus aquaticus. Journ. linn. Soc. Zool. London, 34, 335-343.

VANDEL, A. (1943) - Essai sur l'origine, l'évolution et la classification des Oniscoidea (Isopodes terrestres). Bull. biol. France Belgique, Suppl. 30, 1-136.

- (1947) - Recherches sur la génétique et la sexualité des Isopodes terresres X. Bull. biol. France-Belgique, 81, 1/2, 154-176.

- (1964) - Biospéologie - La biologie des animaux cavernicoles. GauthierVillars, Paris, 1-619.

Van EMden, F. (1922) - Zur Kenntnis der Brutpflege von Asellus aquaticus nebst einigen Bemerkungen über die Brutpflege anderer Isopoden. Arch. $f$. Naturgesch. Berlin, 88, 1, 91-133.

\section{EXPLICATIONS DES PLANCHES 111 (1) - 112 (2)}

Planche 111 (1): 1. Mue postérieure de Stenasellus virei adulte. Elle est composée des 3 derniers péréionites et du pléotelson, avec tous leurs appendices. La mue est blanchâtre et translucide, mais les portions constituées de nombreuses couches cuticulaires superposées, au niveau des pléopodes, sont plus opaques. On voit que la rupture s'est produite au niveau de la membrane articulaire, entre les péréionites IV et V. - 2. Mue antérieure de Stenasellus virei. L'exuviation survient 8 à 16 jours après la perte de l'exuvie postérieure, pour les spécimens étudiés. La mue comporte le céphalon et les 4 péréionites antérieurs, avec leurs appendices. Elle est, en général, plus chiffonnée que l'échantillon photographié.

Planche 112 (2): A. Péréiopode VII droit anormalement régénéré d'un mâle adulte du gouffre de Padirac (Lot). m: moignon du membre amputé dans la partie distale de son basipodite; f: partie du régénérat simulant un pléopode I $\sigma^{*}$ de St. virei; $\mathrm{e}=200$ microns. - B. Propodite et dactylopodite du péréiopode VII gauche du même mâle, à une échelle identique. Les St. virei de Padirac constituent une souche à 2 épines dactyliennes sternales. - C. Pléopode II droit d'une femelle juvénile de $5,5 \mathrm{~mm}$ de Couflens de Betmajou (Ariegè), présentant une duplicature, vraisemblablement à la suite d'une blessure ou amputation en cours de mue; $\mathrm{e}=100$ microns. 
2
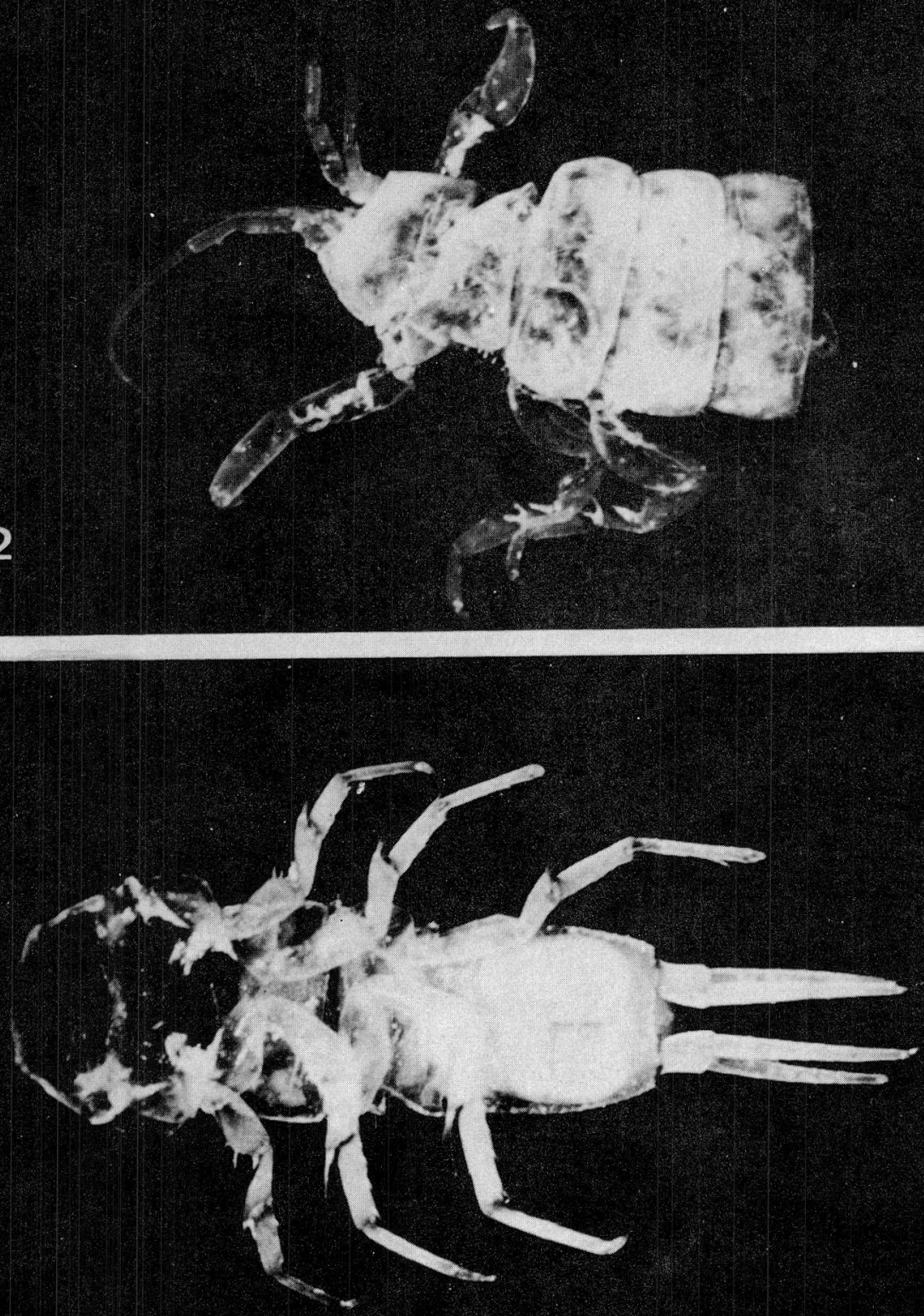
SPELEOLOGY III

(Magniez, 2)

PLATE 112

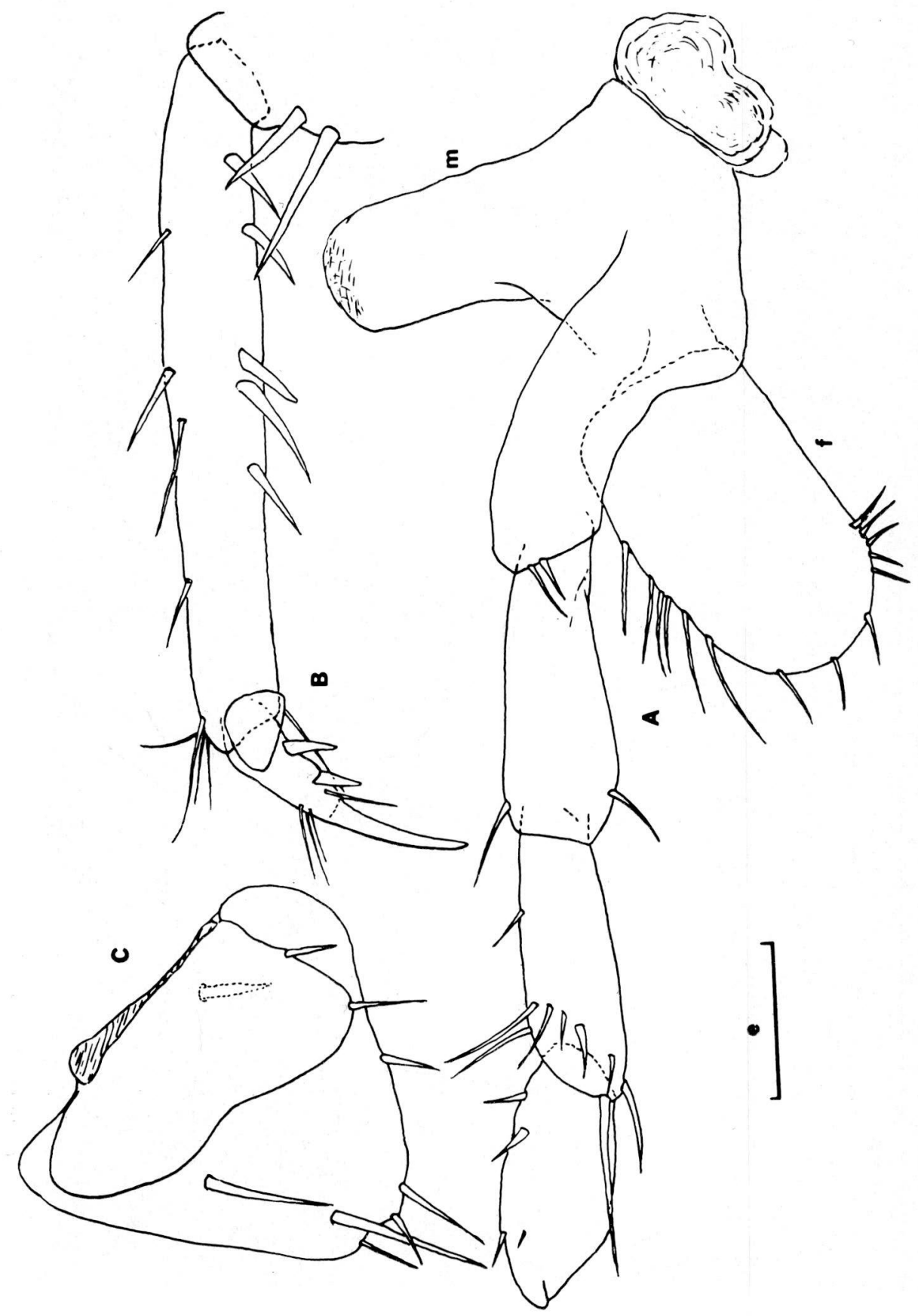

